\title{
F-18-Fluorodeoxyglucose (FDG) positron-emission tomography of Echinococcus multilocularis liver lesions: prospective evaluation of its value for diagnosis and follow-up during benzimidazole therapy
}

\author{
Stumpe, K D M ; Renner-Schneiter, E C ; Kuenzle, A K ; Grimm, F ; Kadry, Z ; Clavien, P A ; \\ Deplazes, P ; von Schulthess, G K ; Muellhaupt, B ; Ammann, R W ; Renner, E L
}

\begin{abstract}
Background:: Long-term benzimidazole therapy benefits patients with non-resectable alveolar echinococcosis (AE). Methods to assess early therapeutic efficacy are lacking. Recently, AE liver lesions were reported to exhibit increased F-18-fluorodeoxyglucose (FDG) uptake in positron emission tomography (PET). To assess the value of FDG-PET for diagnosis and follow-up of AE patients. Patients/Methods:: Twenty-six consecutive patients with newly diagnosed AE were enrolled. Baseline evaluation included CT and FDG-PET. Thirteen patients (11 women; median age 50 years, range 40-76) were resected, the remaining 13 ( 8 women; median age 60 years, range 39-72) had non-resectable disease, were started on benzimidazoles, and CT and FDG-PET were repeated at 6, 12 and 24 months of therapy. Twelve consecutive patients with newly diagnosed cystic echinococcosis (CE) of the liver were also subjected to baseline FDG-PET. Results:: In 21/26 AE patients, baseline PET scans showed multifocally increased FDG uptake in the hepatic lesions' periphery, while liver lesions were FDG negative in 11/12 CE patients. Thus, sensitivity and specificity of FDG-PET for AE vs. CE were $81 \%$ and $92 \%$, respectively. In 5 of 10 non-resectable patients with increased baseline FDG uptake, the intensity of uptake decreased (or disappeared) during benzimidazole therapy, in 3 by 2 grades within the initial 6 months. Conclusions:: FDG-PET is a sensitive and specific adjunct in the diagnosis of suspected AE and can help in differentiating AE from CE. The rapid improvement of positive PET scans with benzimidazole therapy in some patients indicates that absent FDG uptake does not necessarily reflect parasite viability
\end{abstract}

DOI: https://doi.org/10.1007/s15010-007-6133-9

Posted at the Zurich Open Repository and Archive, University of Zurich

ZORA URL: https://doi.org/10.5167/uzh-155760

Journal Article

Published Version

Originally published at:

Stumpe, K D M; Renner-Schneiter, E C; Kuenzle, A K; Grimm, F; Kadry, Z; Clavien, P A; Deplazes, P; von Schulthess, G K; Muellhaupt, B; Ammann, R W; Renner, E L (2007). F-18-Fluorodeoxyglucose (FDG) positron-emission tomography of Echinococcus multilocularis liver lesions: prospective evaluation of its value for diagnosis and follow-up during benzimidazole therapy. Infection, 35(1):11-18.

DOI: https://doi.org/10.1007/s15010-007-6133-9 


\title{
F-18-Fluorodeoxyglucose (FDG) Positron-Emission Tomography of Echinococcus multilocularis Liver Lesions: Prospective Evaluation of its Value for Diagnosis and Follow-up during Benzimidazole Therapy
}

\author{
K. D. M. Stumpe, E. C. Renner-Schneiter, A. K. Kuenzle, F. Grimm, Z. Kadry, P.-A. Clavien, \\ P. Deplazes, G. K. von Schulthess, B. Muellhaupt, R. W. Ammann, E. L. Renner
}

\begin{abstract}
Background: Long-term benzimidazole therapy benefits patients with non-resectable alveolar echinococcosis (AE). Methods to assess early therapeutic efficacy are lacking. Recently, AE liver lesions were reported to exhibit increased F-18-fluorodeoxyglucose (FDG) uptake in positron emission tomography (PET). To assess the value of FDG-PET for diagnosis and follow-up of AE patients.

Patients/Methods: Twenty-six consecutive patients with newly diagnosed AE were enrolled. Baseline evaluation included CT and FDG-PET. Thirteen patients (11 women; median age 50 years, range 40-76) were resected, the remaining 13 ( 8 women; median age 60 years, range 39-72) had non-resectable disease, were started on benzimidazoles, and CT and FDG-PET were repeated at 6, 12 and 24 months of therapy. Twelve consecutive patients with newly diagnosed cystic echinococcosis (CE) of the liver were also subjected to baseline FDG-PET.

Results: In 21/26 AE patients, baseline PET scans showed multifocally increased FDG uptake in the hepatic lesions' periphery, while liver lesions were FDG negative in 11/12 CE patients. Thus, sensitivity and specificity of FDG-PET for $A E$ vs. CE were $81 \%$ and $92 \%$, respectively. In 5 of 10 non-resectable patients with increased baseline FDG uptake, the intensity of uptake decreased (or disappeared) during benzimidazole therapy, in 3 by $\geq 2$ grades within the initial 6 months.

Conclusions: FDG-PET is a sensitive and specific adjunct in the diagnosis of suspected $A E$ and can help in differentiating $A E$ from CE. The rapid improvement of positive PET scans with benzimidazole therapy in some patients indicates that absent FDG uptake does not necessarily reflect parasite viability.
\end{abstract}

Infection 2007; 35: 11-18

DOI 10.1007/s15010-007-6133-9

\begin{tabular}{ll}
\multicolumn{2}{c}{ Abbreviations } \\
E. multilocularis & Echinococcus multilocularis \\
E. granulosus & Echinococcus granulosus \\
AE & alveolar echinococcosis \\
CE & cystic echinococcosis \\
PET & positron emission tomography \\
FDG & fluorodeoxyglucose
\end{tabular}

Introduction

The larval stage of the fox tapeworm Echinococcus multilocularis (E. multicocularis) causes in humans alveolar echinococcosis (AE), a disease primarily affecting the liver. E. multilocularis is prevalent throughout the northern hemisphere [1-3]. An annual incidence of AE of 0.2-1.4 per 100,000 population has been estimated in central Europe $[2,4]$.

\footnotetext{
K. D. M. Stumpe, A. K. Kuenzle, G. K. von Schulthess

Clinic of Nuclear Medicine, University Hospital Zurich, Zurich, Switzerland E. C. Renner-Schneiter, B. Muellhaupt, R. W. Ammann, E. L. Renner Clinic of Gastroenterology and Hepatology, University Hospital Zurich, Zurich, Switzerland

F. Grimm, P. Deplazes

Institute of Parasitology, University of Zurich, Zurich, Switzerland

Z. Kadry, P.-A. Clavien

Dept. of Visceral and Transplantation Surgery, University Hospital Zurich, Zurich, Switzerland

Z. Kadry

Division of Transplantation, Department of Surgery,

The Milton S., Hershey Medical Center, Hershey, PA, USA

E. C. Renner-Schneiter, E. L. Renner (corresponding author)

Dept. of Medicine/Section of Hepatology, University of Manitoba, John Buhler Research Centre, Room 809-715 McDermott Avenue, Winnipeg, MB R3A 1R9, Canada; e-mail: rennerel@cc.UManitoba.CA

K. D. M. Stumpe and E. C. Renner-Schneiter contributed equally to this work.
}

Received: May 19, 2006 • Revision accepted: October 24, 2006 
Biologically, E. multicocularis behaves in the liver like a slowly, but invasively and destructively growing malignant tumor. Without treatment, $>90 \%$ of patients die within 10 years of diagnosis $[1,2,5]$. Radical resection is the treatment of choice $[3,5,6]$. At diagnosis, however, only one third of AE lesions are still amenable to radical resection [4]. Long-term treatment with the benzimidazoles Mebendazole or Albendazole reduces 10-year mortality in non-resectable cases to less than $20 \%[2,5]$. While it is accepted that benzimidazoles are parasitostatic, i.e. inhibiting disease progression, the controversy remains unsettled whether benzimidazoles may be parasitocidal, i.e. curative, and may therefore safely be stopped after years of treatment in some patients. In a previously reported series of non-resectable AE, about one third of patients relapsed upon stopping long-term benzimidazole treatment, while the other two thirds did not [7,8]. Moreover, parasite samples obtained from patients with long-lasting benzimidazole therapy proved still viable in an in vitro assay [5, 9]. Unfortunately, no clinical, laboratory or imaging marker has been identified for assessing the early therapeutic efficacy of benzimidazole therapy. This is largely due to the difficulty of predicting the parasite's viability in vivo.

In contrast to alveolar echinococcosis, treatment of cystic echinococcosis with an expanding growth and usually no invasion and destruction by cysts, should be reserved for symptomatic lesions or those affecting vital anatomical structures [3]. There have been no major clinical trials for any treatment modality in patients with cystic echinococcosis.

E. multilocularis liver lesions were reported to show increased FDG (fluorodeoxyglucose) uptake in PET imaging, possibly reflecting parasite viability $[10,11]$. The purpose of this prospective study was therefore to determine the sensitivity and specificity of FDG-PET in the diagnosis of E. multilocularis liver lesions and to explore the evolution of FDG avid lesions during benzimidazole treatment.

\section{Patients and Methods}

The study was performed according to the declaration of Helsinki. The protocol was approved by the hospital ethics committee and written informed consent was obtained from all participants.

\section{Patients with Alveolar Echinococcosis}

From July 2000 to December 2004, patients (both genders, age 18 to 80 years) with AE were eligible for the study, provided they fulfilled the following criteria: a) new diagnosis without previous treatment or benzimidazole therapy for less than 1 month or b) new diagnosis of recurrence after attempted radical surgery or c) progressive disease, despite benzimidazole therapy.

Diagnosis of AE was based on morphological imaging studies (CT or MRI), serological results and/or histology, as defined by WHO [1]. Serum antibodies against Echinococcus antigens were determined by ELISA, as described [8].

At study entry, a contrast-enhanced CT was performed using a Siemens CT scanner (SomatomPlus 4, Siemens AG, Erlangen, Germany). Spiral CT sections of the neck, thorax, abdomen and pelvis were obtained according to the guidelines of the American College of Radiology. All E. multilocularis lesions were classified according to the PNM system describing the anatomical localisa- tion and extent of $\mathrm{AE}$, i.e. hepatic localisation/size of the parasite $(\mathrm{P})$, and absence/presence of involvement of neighbouring organs $(N)$ and distant metastases $(M)[6,12]$. In addition, the presence/absence of calcifications of E. multilocularis lesions was assessed.

A total of 32 patients with AE were referred to our Echinococcosis Clinic. Twenty six patients fulfilled the inclusion criteria, while 6 were excluded for the following reasons: referral after radical resection, only $(\mathrm{n}=3)$; benzimidazole therapy for more than 1 month prior to referral $(\mathrm{n}=1)$; age less than 18 years $(\mathrm{n}=1)$ or more than 80 years $(\mathrm{n}=1)$, respectively.

Apart from echinococcosis, none of the AE patients presented evidence of active infection, except for one patient (Table 1, \#14) who had HIV infection that was well controlled by antiretroviral therapy [13].

\section{Patients with Cystic Echinococcosis}

In order to define the specificity of FDG-PET for EM liver lesions, 12 consecutive patients with Echinococcus granulosus (E. granulosus), the dog tapeworm, causing cystic echinococcosis (CE), also underwent FDG-PET studies. They fulfilled the following criteria: a) newly diagnosed disease without previous treatment $(n=6)$ or benzimidazole therapy for less than two weeks $(\mathrm{n}=3)$, or b) recurrence after radical surgery treated with benzimidazole therapy for less than two weeks $(n=3)$. Diagnosis of $\mathrm{CE}$ was based on morphological imaging studies (CT or MRI) and serological results as defined by WHO [1]. In one of these patients serology and further work-up revealed later no evidence for $\mathrm{CE}$ and the diagnosis of a simple, benign liver cyst was made (Table 2,\#12).

None of the CE patients had evidence of active infections other than echinococcosis, except for one patient (Table 2, \#11) who presented with symptomatic choledocholithiasis and mild cholangitis.

\section{Follow-up Alveolar Echinococcosis}

Thirteen of the 26 patients with AE underwent radical resection followed by a 2 years course of benzimidazole therapy $[1,6]$. In 5 of these 13 patients material from the E. multilocularis lesion was collected intraoperatively and subjected to viability testing using an animal model [9]. Twelve of the 13 resected patients survived and remain recurrence free, one (Table 1,\#9) died from multiorgan failure within the early postoperative period.

In 12 of the 13 non-resectable AE patients long-term benzimidazole therapy was started. One patient (Table 1, \#20) with extensively calcified lesions and without detectable anti-Echinococcus antibodies was followed without therapy. Four hours post-ingestion Albendazol and Mebdenazole serum levels were targeted to $>1 \mathrm{umol} / \mathrm{l}$ and $>250 \mathrm{nmo} / \mathrm{l}$, respectively $[5,8]$.

Non-resectable AE patients were followed with physical exam, lab tests and imaging studies including CT and FDG-PET. One patient died 2 months after baseline examination from metastatic renal cell carcinoma (Tabel 1, \#18).

\section{Viability Testing}

In vivo viability testing was performed in jirds (Meriones unguiculatus), as described [9]. In brief, $0.25 \mathrm{ml}$ homogenate prepared in physiological saline from an intraoperatively collected portion of the E. multilocularis resection specimen was intraperitoneally injected in jirds (3-5 animals per specimen). After four to six months, animals were sacrificed and the abdomen macro- and microscopically examined for metacestode proliferation.

\section{PET and PET/CT}

A single baseline FDG-PET examination was performed in the patients with $\mathrm{CE}(\mathrm{n}=12)$ and in the patients with resectable AE 
$(\mathrm{n}=13)$. The non-resectable AE patients $(\mathrm{n}=13)$ received a baseline FDG-PET examination and those surviving $(n=12)$ were followed by FDG-PET at 6, 12 and 24 months, respectively, or until $\geq 2$ consecutive scans were negative.

Initially FDG-PET and CT examinations were performed separately on the same day. Later-on, an integrated PET/CT device became available and PET examinations were performed using this new scanner.

\section{PET-Imaging Protocol}

FDG-PET-studies were performed on a GE Advance PET scanner (GE Medical Systems, Waukesha, Wis., USA). The scanner acquires $352 \mathrm{D}$ sections of $4.25 \mathrm{~mm}$ thickness per increment with an axial field of view of $14.6 \mathrm{~cm}$. The patients were explicitly asked to fast for at least 4 hours prior to the study. Fifty minutes prior to emission scanning, the patients received an intravenous injection of 300 to $400 \mathrm{MBq}$ of FDG. Image reconstruction was performed with a multiplicative iterative reconstruction algorithm for improvement of image quality and reduction of computation time [14].

$\mathrm{PET} / \mathrm{CT}$ studies were performed on an integrated PET/CT device (Discovery LS, GE medical systems, Waukesha, Wisconsin, USA), combining the ability to acquire CT-images and PET data of the same patient in one session. A GE Advance NXi PET scanner and a four-slice CT (LightSpeed plus) were integrated in this dedicated system. An unenhanced low dose CT was performed from the patient's head to the pelvic floor.

\section{PET and PET/CT Image Evaluation}

The PET scans were analysed by two board certified nuclear physicians with extended experience, first separately and then in consensus according to the procedure described below. They were blinded to clinical data, the results of laboratory and other imaging studies, as well as to the final diagnosis.

Images were visually analysed as follows. First, the readers analysed the attenuation-corrected PET images of every patient. Then, if available, the CT images, attenuation-corrected PET images, and co-registered PET/CT images were displayed together on the monitor, and the reviewers again evaluated the images. Physiological FDG uptake was identified, and excluded from further analysis.

In a second step, the lesions deemed to be pathological were classified according to a semi-quantitative FDG-uptake grading scale from 0 to 4 : $0=$ uptake as in the background, $1=$ low uptake comparable to uptake in inactive muscles and fat, $2=$ moderate uptake clearly noticeable and distinctly higher than uptake into inactive muscles and fat, 3 = strong uptake, but distinctly below FDG uptake noted in normal cerebral cortex, $4=$ very strong uptake/comparable to normal cerebral cortex. This grading scale was adapted from Stumpe et al $[15,16]$. It has been shown that classifying grade 3 and 4 lesions as infectious foci yields the best discrimination to non-infectious lesions. The presence or absence of the following was also recorded: Localization and size of the lesion, the extension/invasion into adjacent organs, the uptake pattern (diffuse, circular or focal) and whether there was metastatic spread into distant organs.

AE was diagnosed in PET if pericystic FDG uptake was visible according to Reuter et al. According to our PET experience and the clinical features of $\mathrm{AE}$ and $\mathrm{CE}$, the diagnosis of $\mathrm{CE}$ was made if no FDG uptake was present around the cyst.

\section{Statistics}

Data are given as median and range. Mann-Whitney $U$ or Fisher's exact test were used for group comparison, as appropriate. A p value (two-sided) $\leq 0.05$ was considered statistically significant.

\section{Results}

Baseline

Patients with Alveolar Echinococcosis (AE)

Twenty-three of the 26 patients had newly diagnosed disease (Table 1), whereas 3 had AE recurrence after attempted radical resection and/or cessation of long-term benzimidazole therapy. Thirteen patients underwent radical resection, the remaining 13 presented with non-resectable lesions. There were no significant differences in age and gender distribution between resectable and non-resectable cases. As expected, more patients in the resectable than in the non-resectable group had stage I-IIIa, i.e. less advanced, disease $(\mathrm{p}=0.047)$.

Increased FDG uptake was observed in 21 of the 26 patients, i.e. in 11 of 13 with resectable and in 10 of 13 patients with non-resectable AE, respectively ( $\mathrm{p}=$ n.s.). A typical example of increased perilesional FDG uptake in a patient with a large E. multilocularis mass in the right liver lobe (Table 1, \#17) is depicted in Fig. 1. All positive cases showed focally increased FDG uptake in the lesion's periphery. In eighteen of the 21 positive PET scans, the intensity of FDG uptake was graded $\geq 3$, while it was graded 2 in the remaining 3 patients. Five cases were PET negative, two of them (Table 1, \#20 and \#7) presenting with extensively calcified lesions. Overall, sensitivity of FDG-PET for AE was $81 \%(21 / 26)$.

Viability testing of E. multilocularis tissue removed at surgery was performed in 7 of the 13 resectable AE patients. Two of the 7 patients had PET negative lesions and viability testing was negative in both (Table 1, \#7 and \#11). However, only 1 of the 5 viability tests performed with material of PET positive resection specimens yielded a positive result (Table 1, \#12), the other four remained negative (Table 1, \#1, \#2, \#13 and \#9).

\section{Patients with Cystic Echinococcosis (CE)}

Except for one patient, all CE cases were PET negative (Table 2). Thus, specificity of FDG-PET was $92 \%(11 / 12)$ in this series. Moreover, the sole positive CE patient (Table 2 , \#11) was only weakly positive (grade 2) and presented with symptomatic choledocholithiasis. Thus, some cholangitis-related weak PET positivity cannot be ruled out.

\section{Follow-up of Non-resectable AE during Benzimidazole Therapy}

All non-resectable AE patients were started on longterm benzimidazole therapy, except for one patient without detectable anti-Echinococcus antibody levels (EmII/3-10 and Em2-plus-ELISA) and with an extensively calcified, PET negative lesion that was deemed avital (Table 1, \#20). He was followed without treatment. One patient died from metastatic renal cell carcinoma before the scheduled follow-up PET (Table 1,\#18). The median $4 \mathrm{~h}$ post ingestion serum level of 


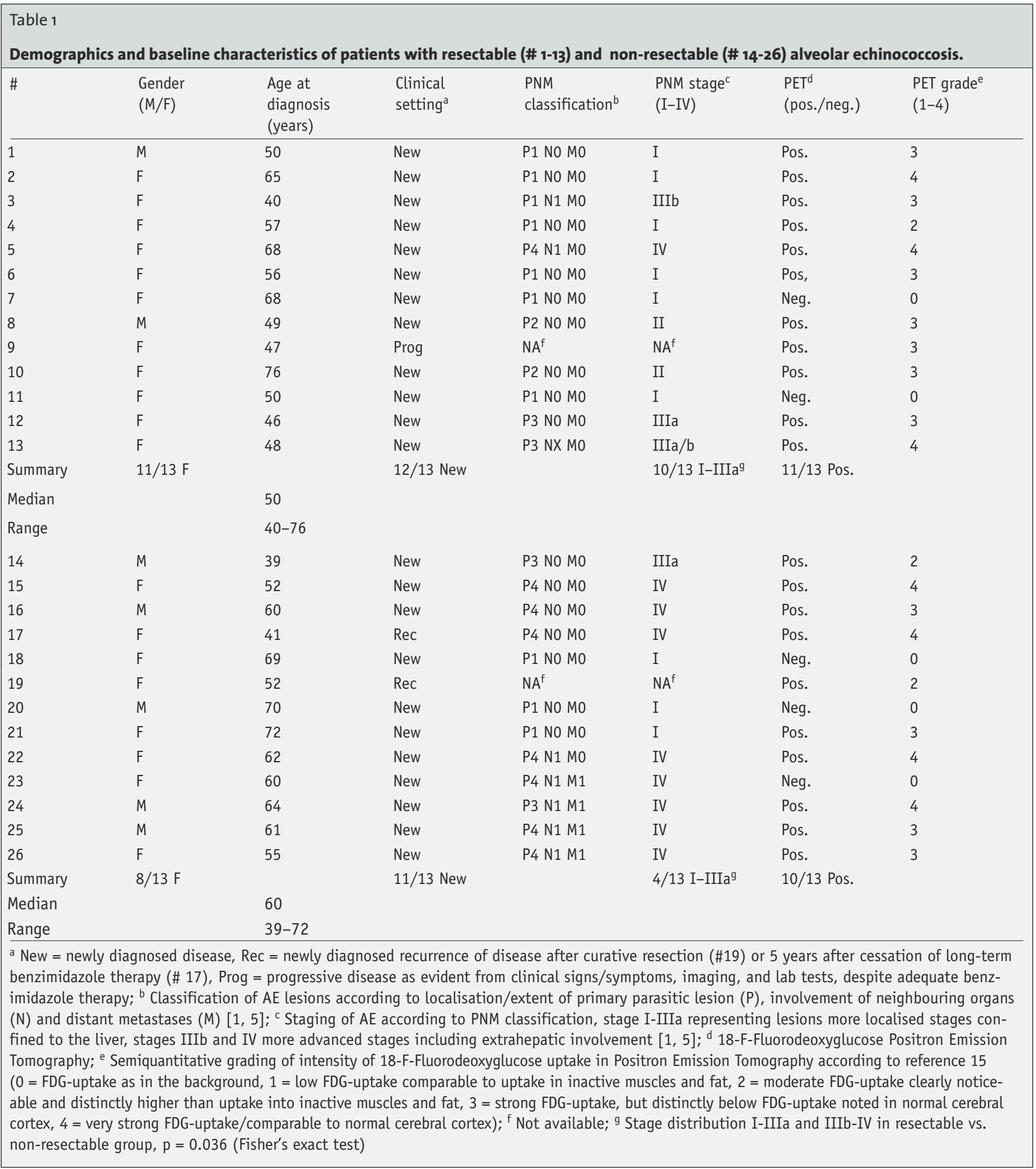




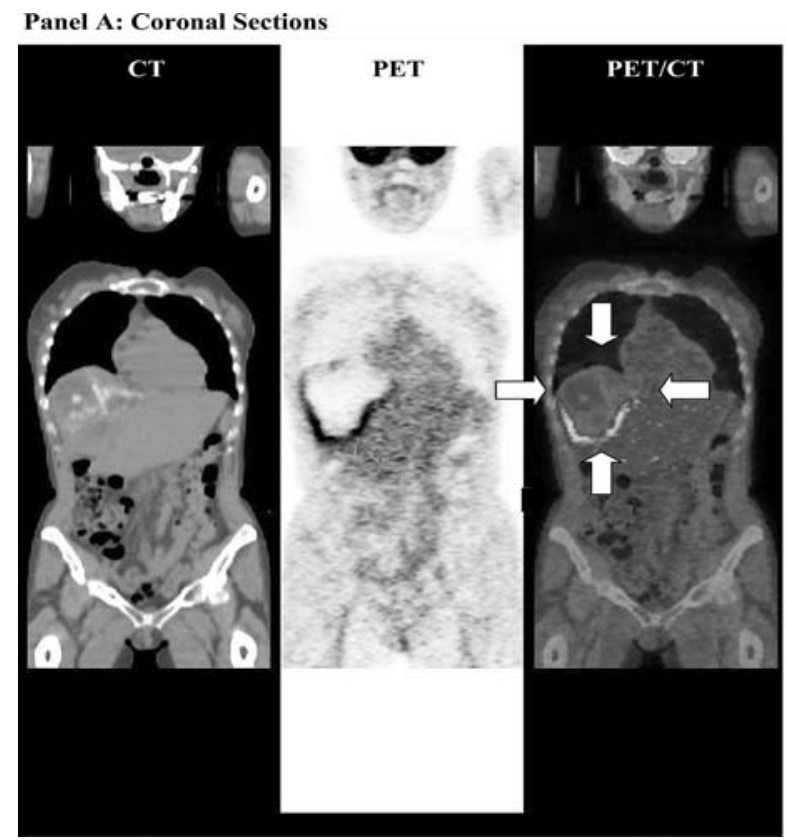

Panel B: Transverse Sections

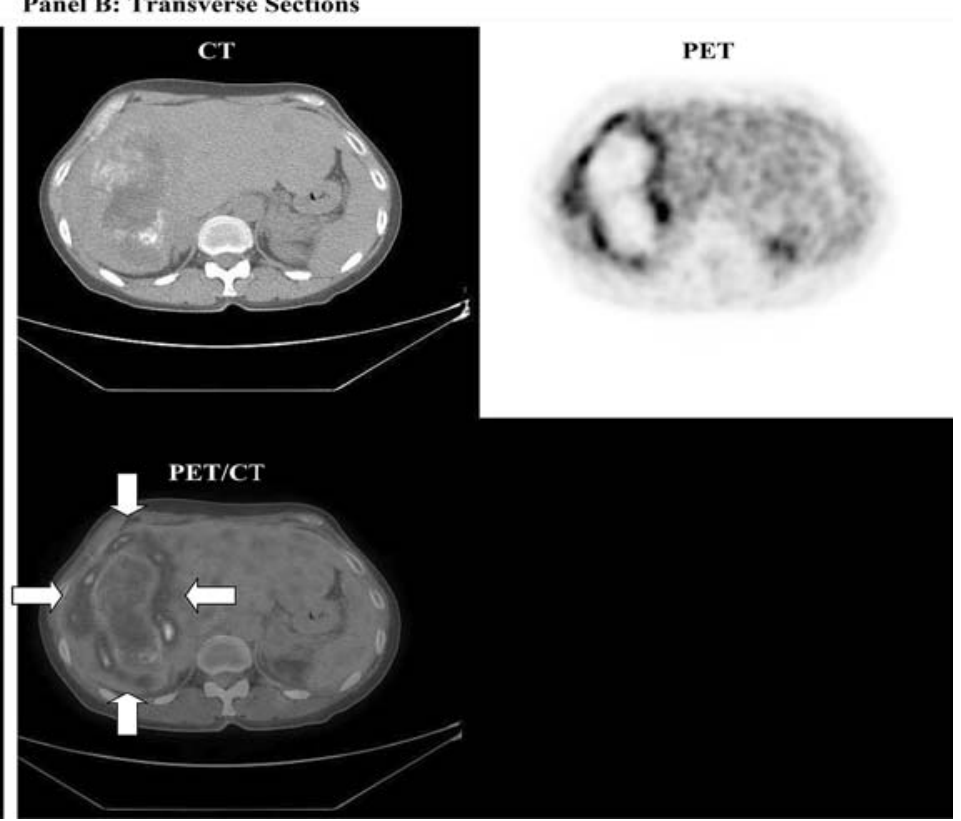

Figure 1. Depicted is an example of coronal sections (Panel A) and transverse sections (Panel B) of a large cystic lesion in the right liver lobe (arrows) in a patient with alveolar echinococcosis (Table 1, \#17), as assessed by computerized tomography (CT), fluorodeoxyglucose (FDG)positron emission tomography (PET), and combined PET/CT. Note the multifocally increased FDG uptake in the periphery of the lesion (arrows), while its centre exhibits irregular calcifactions on CT and does not take up FDG in PET, compatible with extensive necrosis.

albendazole was $4.2 \mathrm{umol} / \mathrm{l}$ (range 0.6-14.9), that of mebendazole $261 \mathrm{nmol} / 1(50-1689)$ respectively.

In 5 of 10 non-resectable patients with positive FDGPET at baseline, PET positivity on follow-up scans had decreased in intensity grade $(\mathrm{n}=1)$ or disappeared completely $(\mathrm{n}=4)$ after 24 months of benzimidazole therapy. In 3 of these 5 patients, FDG uptake decreased by $\geq 2$ grades within 6 months of starting benzimidazoles. On the other hand, PET positivity remained stable in $2 / 10$ and increased in $3 / 10$ patients, respectively. None of the two surviving baseline PET negative patients became PET positive during follow-up.

Benzimidazole drug levels did not significantly differ in patients with and without improvement or loss of PET positivity, median $4 \mathrm{~h}$ post ingestion levels of albendazole amounting to $3.6 \mathrm{umol} / \mathrm{l}(0.6-7.5)$ and $4.6(1.9-14.9)$, those of mebendazole to $116 \mathrm{nmol} / 1$ (50-261) and 299 (140-1689), respectively (both $\mathrm{p}=\mathrm{ns}$ ). Comparison of the baseline characteristics of the 5 patients with improvement/loss of PET positivity to those of the 5 patients without revealed no significant differences in gender, age, PNM stage, specific anti-Echinococcus antibody levels, or local extension and/or intensity grade of baseline PET positivity (data not shown).

\section{Discussion}

Recently, Reuter et al. reported that E. multilocularis liver lesions may show FDG uptake and thus be visualized with PET [10]. It was suggested that a positive FDG-PET scan may reflect parasite viability in vivo and thus may potentially be useful in deciding on stopping long-term benzimidazole therapy in some non-resectable cases [11]. However, interpretation of these data is difficult since a limited number of patients with a rather heterogeneous disease history was studied, including newly diagnosed cases and patients treated for various times with benzimidazoles.

Our study demonstrates a high diagnostic sensitivity and specificity of FDG-PET in differentiating CE from AE which may be difficult by conventional imaging modalities and serology alone. Thus, FDG-PET represents a valuable diagnostic adjunct in suspected hepatic echinococcosis.

The lack of perilesionally increased FDG uptake in CE would be compatible with a smaller mass of glucose metabolizing parasitic tissue or with a lower rate of glucose metabolism in CE compared to AE. Alternatively, a differing reaction of the host to the parasite may be involved. The morphology of the parasite-host relationship differs markedly between $\mathrm{CE}$ and $\mathrm{AE}[1,17,18]$. CE lesions are characterized by a broad homogeneous capsule composed of laminated parasite and perilesional fibrotic host tissue, whereas E. multilocularis grows multifocally and invasively with protrusions of its germinal layer into the host tissue causing an intense inflammatory host reaction.

Increased FDG uptake has been observed in tumoral and inflammatory/infectious lesions and a decrease in FDG uptake is considered a useful parameter in assessing the effect of anti-tumoral and anti-infectious pharmacotherapy [19]. The parasitic proliferation in AE occurs typically in 


\begin{tabular}{|c|c|c|c|c|c|}
\hline \# & $\begin{array}{l}\text { Gender } \\
(M / F)\end{array}$ & $\begin{array}{l}\text { Age at diagnosis } \\
\text { (years) }\end{array}$ & Final diagnosis & $\begin{array}{l}\text { PET a } \\
\text { (pos./neg.) }\end{array}$ & $\begin{array}{l}\text { PET Grade } \\
(1-4)\end{array}$ \\
\hline 1 & $M$ & 45 & Cystic echinococcosis & Neg. & 0 \\
\hline 2 & $\mathrm{~F}$ & 48 & Cystic echinococcosis & Neg. & 0 \\
\hline 3 & $M$ & 42 & Cystic echinococcosis & Neg. & 0 \\
\hline 4 & $M$ & 25 & Cystic echinococcosis & Neg. & 0 \\
\hline 5 & $M$ & 42 & Cystic echinococcosis & Neg. & o \\
\hline 6 & $M$ & 22 & Cystic echinococcosis & Neg. & o \\
\hline 7 & $\mathrm{~F}$ & 60 & Cystic echinococcosis & Neg. & 0 \\
\hline 8 & $M$ & 42 & Cystic echinococcosis & Neg. & 0 \\
\hline 9 & $\mathrm{~F}$ & 32 & Cystic echinococcosis & Neg. & o \\
\hline 10 & $\mathrm{~F}$ & 40 & Cystic echinococcosis & Neg. & 0 \\
\hline 11 & $\mathrm{~F}$ & 67 & Cystic echinococcosis & Pos. ${ }^{c}$ & 2 \\
\hline 12 & $M$ & 69 & $\begin{array}{l}\text { Simple Benign Liver } \\
\text { Cyst }^{d}\end{array}$ & Neg. & 0 \\
\hline Summary & $5 / 12 \mathrm{~F}$ & & & 11/12 Neg. & \\
\hline Median & & 42 & & & \\
\hline Range & & $22-69$ & & & \\
\hline \multicolumn{6}{|c|}{ 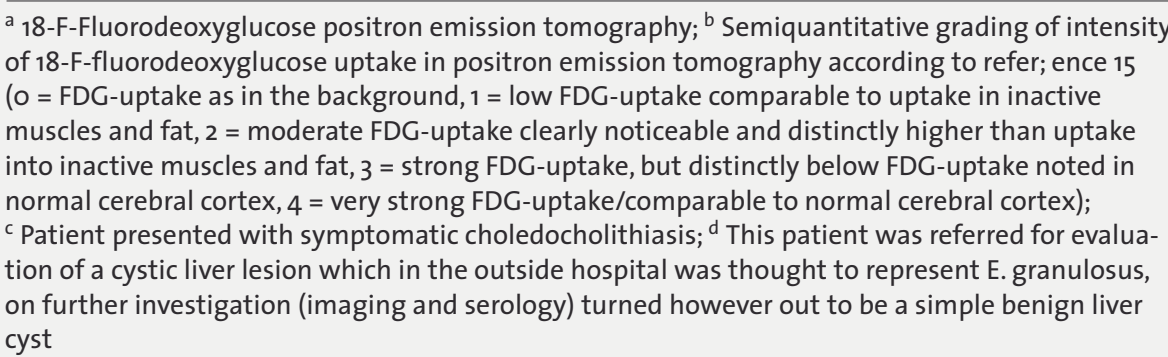 } \\
\hline
\end{tabular}

foci at the periphery of lesions where also increased FDG uptake is located. FDG uptake is absent in the centre of AE lesions where mostly necrotic material is found.

Interestingly, the decrease in intensity or complete loss of FDG uptake observed during benzimidazole therapy in 5 out of 10 patients with non-resectable AE occurred rapidly, often within the first 6 months of treatment. This makes a decrease/loss of FDG uptake unlikely to reflect death of the parasite. Thus, in a previous study AE recurrence was observed after stopping long-term (average 4.3 years) mebendazole therapy in 7 of 19 patients within 6-24 months, proving that the parasite was still vital in $1 / 3$ of patients after years of benzimidazole treatment [7].

At present it seems not possible to attribute the increased FDG uptake in the periphery of AE lesions to parasitic metabolic activity and/or vitality, perilesional inflammation, or a combination of both.

The issue of FDG uptake and parasite vitality has to be discussed also in the context of the $5 \mathrm{AE}$ patients with negative baseline PET studies. It is tempting to speculate that these patients may have had so called "died-out" AE lesions. Spontaneous inactivation of AE lesions has been documented [20,21] and recently reviewed $[1,2]$. The following supports that the AE lesions in 4 of the 5 patients with negative baseline FDG-PET were, indeed, "died-out": material from $\mathrm{AE}$ lesions of both resected patients tested negative in the in vivo viability assay (Table 1, \#7 and \#11); moreover, in 2 patients with non-resectable AE lesions (Table 1, \#20 and \#23) follow-up examinations were fully compatible with died out lesions, i.e. were extensively calcified and/or remained stable and PET negative, in one of them (Table 1, \#20) even in the absence of benzimidazole therapy. The third patient with a PET negative AE lesion at baseline died from metastatic renal cell carcinoma prior to the 6 months follow-up examination (Table 1, \#18). While the lack of FDG uptake at baseline may have truly reflected avitality of the parasite in at least 4 of the 5 patients, alternative explanations for PET negative lesions can not entirely be ruled out: a) the metabolic activity of the parasite may for some unexplained reason(s) have been spontaneously too low to be picked up by PET and/or b) PET positivity of very small peripheral foci may have been missed due to the limited spatial resolution of the PET scanner $(5 \mathrm{~mm})$.

Material obtained at resection from only 1 of 5 PET positive patients tested positive in the in vivo viability assay (Table 1,\#12). The sensitivity of this assay in untreated $\mathrm{AE}$ cases has been reported to be $80 \%$ [9]. EM is known to grow and proliferate in discrete foci at the lesions periphery, only. The low percentage of positive viability tests $(20 \%)$ in the current series may be attributable to sampling error introduced by failing to systematically random sample the entire periphery of the resected specimen. Thus, the material subjected to viability testing may have been collected by chance from a portion of the resection specimen not containing viable parasite.

Our study complements the reports by Reuter et al. $[10,11]$. In follow-up of their earlier work [10], these 


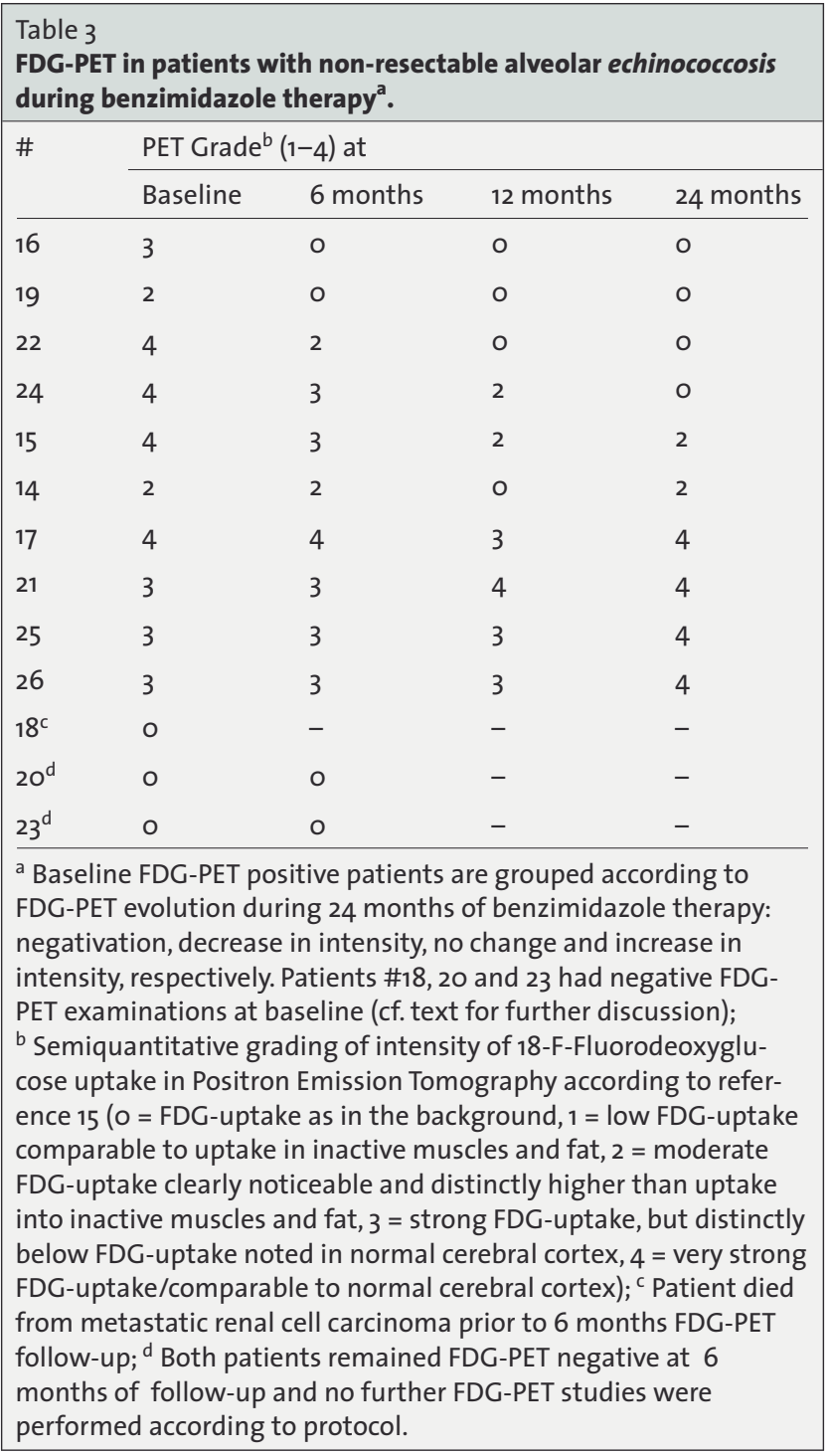

authors discontinued benzimidazole therapy in 15 patients who exhibited PET negative lesions after an average of 5 years of benzimidazole treatment [11]. After a further 18 months off therapy, 8 of the 15 patients showed progression of the disease defined as either newly developed PET positivity $(n=6)$ or clinical signs of progression $(\mathrm{n}=2)$. This further underscores that PET negativity does not necessarily indicate avitality of the parasite.

In conclusion, our study suggests that FDG-PET/(CT) is a sensitive and specific adjunct to the diagnostic armamentarium in suspected AE and can help in differentiating AE from $\mathrm{CE}$. The rapid improvement of positive FDG-PET scans with benzimidazole therapy indicates that absent FDG uptake does not necessarily reflect parasite vitality. A better understanding of the factors linking parasite metabolism and vitality and/or inflammatory host responses to PET positivity or negativity warrants further evaluation.

\section{Acknowledgements}

This study was supported by the Foundation for Medical Research and Development (Winterthur, Switzerland), the Gebert-Ruef Foundation (Zurich, Switzerland), the Baugarten-Foundation (Zurich, Switzerland), the OPO-Foundation (Zurich, Switzerland), the Caritative Foundation Dr. Gerber-ten Bosch (Zurich, Switzerland) and the UBS (Zurich, Switzerland) acting on behalf of a major anonymous sponsor. We thank our nursing and technical staff and, in particular, our study nurse Karin Riederer, for their meticulous help in conducting this study.

\section{References}

1. Pawlowski ZS, Eckert J, Vuitton DA, Ammann RW, Kern P, Craig P: Echinococcosis in humans: clinical aspects, diagnosis and treatment. In: Eckert J, Gemmell MA, Meslin FX, Pawlowski ZS (eds) WHO/OIE Manual on echinococcosis in humans and animals. World Organisation for Animal Health and WHO, Paris 2001; pp 20-71.

2. Eckert J, Deplazes P: Biological, epidemiological, and clinical aspects of echinococcosis, a zoonosis of increasing concern. Clin Microbiol Rev 2004; 17:107-135.

3. McManus DP, Zhang W, Li J, Bartley PB: Echinococcosis. Lancet 2003; 362:1295-1304.

4. Eckert J, Jacquier P, Baumann D, Raeber PA: Human echinococcosis in Switzerland, 1984-1992. Schweiz Med Wochenschr 1995; 125:1989-1998.

5. Ammann RW, Eckert J: Cestodes. echinococcus. Gastroenterol Clin North Am 1996; 25:655-689.

6. Kadry Z, Renner EC, Bachmann LM, Attigah N, Renner EL, Ammann RW, Clavien PA: Evaluation of treatment and long-term follow-up in patients with hepatic alveolar echinococcosis. $\mathrm{Br} J$ Surg 2005; 92:1110-1116.

7. Ammann RW, Hirsbrunner R, Cotting J, Steiger U, Jacquier P, Eckert J: Recurrence rate after discontinuation of long-term mebendazole therapy in alveolar echinococcosis (preliminary results). Am J Trop Med Hyg 1990; 43:506-515.

8. Ammann RW, Renner EC, Gottstein B, Grimm F, Eckert J, Renner EL: Immunosurveillance of alveolar echinococcosis by specific humoral and cellular immune tests: long-term analysis of the Swiss chemotherapy trial (1976-2001). J Hepatol 2004; 41:551-559.

9. Eckert J, Jacquier P: Viability testing of Echinococcus multilocularis metacestodes from untreated and treated patients. Arch Hidatiol 1991; 30:863-867.

10. Reuter S, Schirrmeister H, Kratzer W, Dreweck C, Reske SN, Kern P: Pericystic metabolic activity in alveolar echinococcosis: assessment and follow-up by positron emission tomography. Clin Inf Dis 1999; 29:1157-1163.

11. Reuter S, Buck A, Manfras B, Kratzer W, Seitz HM, Darge K, Reske SN, Kern P: Structured treatment interruption in patients with alveolar echinococcosis. Hepatology 2004; 39:509-517.

12. Kern P, Wen H, Sato N, Vuitton DA, Gruener B, Shao Y, Delabrousse E, Kratzer W, Bresson-Hadni S: WHO classification of alveolar echinococcosis: principles and application. Parasitol Int 2006; 55(Suppl):S283-287.

13. Zingg W, Renner-Schneiter EC, Pauli-Magnus C, Renner EL, van Overbeck J, Schlapfer E, Weber M, Weber R, Opravil M, Gottstein B, Speck RF: Swiss HIV Cohort Study: Alveolar echinococcosis of the liver in an adult with human immunodeficiency virus type-1 infection. Infection 2004; 32:299-302. 
14. Schmidlin P: Improved iterative image reconstruction using variable projection binning and abbreviated convolution. Eur J Nucl Med 1994; 21:930-936.

15. Stumpe KD, Dazzi H, Schaffner A, von Schulthess GK: Infection imaging using whole-body FDG-PET. Eur J Nucl Med 2000; 27:822-832.

16. Stumpe KD, Notzli HP, Zanetti M, Kamel EM, Hany TF, Gorres GW, von Schulthess GK, Hodler J: FDG PET for differentiation of infection and aseptic loosening in total hip replacements: comparison with conventional radiography and three-phase bone scintigraphy. Radiology 2004; 231:333-341.

17. Bresson-Hadni S, Delabrousse E, Blagosklonov O, Bartholomot B, Koch S, Miguet JP, Andre Mantion G, Angele Vuitton D: Imaging aspects and non-surgical interventional treatment in human alveolar echinococcosis. Parasitol Int 2006; 55(Suppl):S267-272.
18. Vuitton DA, Zhang SL, Yang Y, Godot V, Beurton I, Mantion G, Bresson-Hadni S: Survival strategy of Echinococcus multilocularis in the human host. Parasitol Int 2006; 55(Suppl):S51-55.

19. Zhuang H, Yu JQ, Alavi A: Applications of fluorodeoxyglucosePET imaging in the detection of infection and inflammation and other benign disorders. Radiol Clin North Am 2005; 43: 121-134.

20. Rausch RL, Wilson JF, Schantz PM, McMahon BJ: Spontaneous death of Echinococcus multilocularis: cases diagnosed serologically (by Em2 ELISA) and clinical significance. Am J Trop Med Hyg 1987; 36:576-585.

21. Gottstein B, Saucy F, Deplazes P, Reichen J, Demierre G, Busato A, Zuercher C, Pugin P: Is high prevalence of Echinococcus multilocularis in wild and domestic animals associated with disease incidence in humans? Emerg Inf Dis 2001; 7:408-412. 